\title{
Precision of anterior and posterior corneal curvature measurements taken with the Oculus Pentacam
}

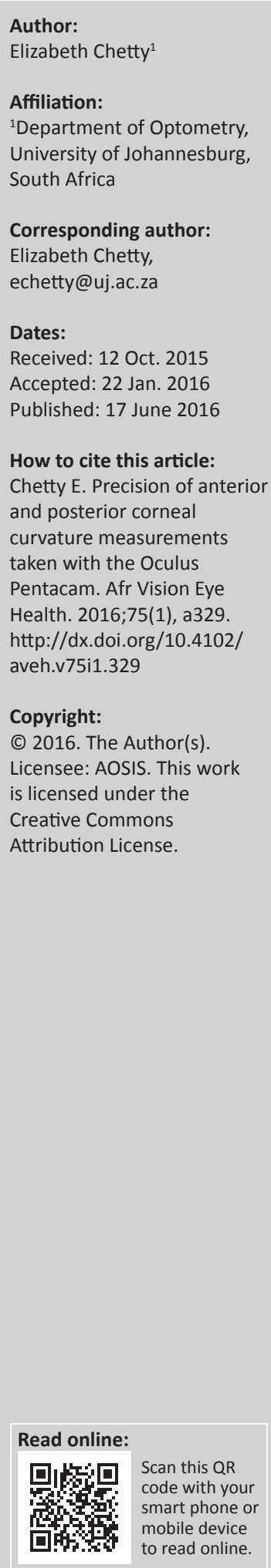

In the era of rapid advances in technology, new ophthalmic instruments are constantly influencing health sciences and necessitating investigations of the accuracy and precision of the new technology. The Oculus Pentacam (70700) has been available for some time now and numerous studies have investigated the precision of some of the parameters that the Pentacam is capable of measuring. Unfortunately some of these studies fall short in confusing the meaning of accuracy and precision and in not being able to analyse the data correctly or completely. The aim of this study was to investigate the precision of the anterior and posterior corneal curvature measurements taken with the Oculus Pentacam (70700) holistically with sound multivariate statistical methods. Twenty successive Pentacam measurements were taken over three different measuring sessions on one subject. Keratometric data for both the anterior and posterior corneal surfaces were analysed using multivariate statistics to determine the precision of the Oculus Pentacam. This instrument was found to have good precision both clinically and statistically for anterior corneal measurements but only good clinical precision for the posterior corneal surface.

\section{Introduction}

According to the International Organization of Standardization (ISO ${ }^{1}$ precision can be defined as 'the closeness of agreement between test results'. McAlinden et al. ${ }^{2}$ further unpack the definition: 'Repeatability and reproducibility are the two faces of precision. Repeatability refers to the variability in repeated measurements by one observer when all other factors are assumed constant. Reproducibility refers to the variability in repeated measurements when one or more factors, such as observer, instrument, calibration, environment, or time is varied'. In this study the repeatability of the Pentacam was investigated by taking a set of 20 successive measurements on one subject and the reproducibility was investigated by taking another two sets of 20 successive measurements on the same subject by the same observer 2 and $4 \mathrm{~h}$ apart from the original set of measurements.

The Pentacam is an anterior eye segment tomographer based on Scheimpflug imaging. It is a dual digital CCD camera system with a UV-free blue LED light source. One camera is stationary and is centred on the pupil to aid fixation and track eye movements and the other camera rotates $360^{\circ}$ taking up to 50 slit images (basic model) in less than $2 \mathrm{~s}$. Data for the whole cornea including both anterior and posterior surfaces is acquired from approximately 138000 true elevation points. . $^{3,4}$ Any eye movements detected with the stationary camera are taken into account when the software analyses the data. The Pentacam is a versatile instrument that provides information on the cornea and anterior chamber, which makes it useful for the detection and tracking of corneal anomalies and glaucoma respectively.

The Pentacam provides a plethora of information on the anterior segment of the eye; however, only the precision of the anterior and posterior corneal keratometric data were considered for the purposes of this study. There are numerous studies that have investigated the precision of the Pentacam in terms of central ${ }^{5,6,7}$ and peripheral ${ }^{8}$ corneal thickness, anterior chamber depth and volume $^{5,6}$ and corneal curvature measurements. ${ }^{9,10,11,12}$ Precision studies are necessary to ensure confidence in the instruments used in the management of patients, especially when it is necessary to refer patients for procedures such as cross linking, for example, and to be able to track the progression of anomalies satisfactorily.

\section{Methods}

This study, as part of a broader investigation of keratometric behaviour, received ethical clearance from the Ethics Committee of the Faculty of Health Sciences of the University of Johannesburg. 
One randomly selected 21-year-old female student volunteer was used for the study. Written informed consent was received after she was briefed on the study. This subject was not on any medication, was free of ocular disease, did not have any previous ocular surgery, and had never worn contact lenses. All measurements were taken $2 \mathrm{~h}$ apart on the same day spread over three measurement sessions which took place at 8 a.m., 10 a.m., and at noon. Each data set consisted of 20 consecutive measurements taken on the right eye of the same subject by the same observer with the Pentacam. The procedure for taking measurements was done according to the Pentacam's user manual, ${ }^{4}$ and after each consecutive measurement the subject was asked to sit back while the instrument was processing to allow for realignment before the next measurement was taken. Each measuring session lasted approximately $10 \mathrm{~min}$.

\section{Statistical analysis}

Many of the previous precision studies done on corneal curvature measurements taken with the Pentacam analysed the keratometric data as two separate components along the principal meridians. Keratometric data is multivariate in nature and should be analysed holistically. This can be achieved by converting the raw keratometric data (radii of curvature along principal meridians) into conventional powers (sphere, cylinder, and axis) which are then converted to dioptric power matrices. Dioptric power matrices are then used to generate stereo-pair scatter plots which provide a visual representation of variation. The methods used to do this are discussed in detail elsewhere. ${ }^{13,14,15,16,17,18,19}$ The statistical software used to analyse this type of data was developed by Harris and Malan, ${ }^{17,18,19}$ with further adaptations by Rubin. This paper included the use of stereo-pair scatter plots and ellipsoidal surfaces of constant probability density (distribution ellipsoids) to aid in the visualisation of variation. Each dot seen on a stereo-pair scatter plot is representative of each transformed Sim-K measurement. Means, variances, and covariances were also calculated and therefore hypothesis testing was possible. The interested reader is referred elsewhere ${ }^{20}$ for a detailed summary of this type of statistical analysis of keratometric data.

\section{Results}

Tables 1 and 2 provide the statistical variables for the keratometric data of the anterior and posterior surfaces of the cornea respectively. Means, variance-covariance matrices and 95\% distribution ellipsoid volumes are provided. The stereo-pair scatter plots below (Figures 1 and 3) are a visual representation of how each keratometric measurement relates to the other and can be viewed three dimensionally with the eyes in an exo-position, that is, with the eyes drifted apart. Keratometric data for all three measuring sessions have been superimposed onto one stereo-pair scatter plot for both the anterior corneal surface (Figure 1) and the posterior corneal surface (Figure 3). For both anterior and posterior corneal surfaces the first measuring session is represented by the black data points, the second session by the red data points, and the third session by the blue data points. Rotated stereo-pair scatter plots (Figures 2 and 4 ) were also generated by rotating the

TABLE 1: Means are included in conventional and component notation with variance-covariance matrices, $95 \%$ distribution ellipsoid volumes for the anterior surface corneal curvature measurements.

\begin{tabular}{|c|c|c|c|c|c|c|}
\hline \multirow{3}{*}{$\begin{array}{l}\text { Session } \\
1 .\end{array}$} & \multicolumn{6}{|c|}{ Anterior surface } \\
\hline & \multirow{2}{*}{$\begin{array}{c}\text { Conventional notation (D) } \\
44.21-1.32 \times 99\end{array}$} & \multirow{2}{*}{$\begin{array}{c}\text { Component notation (D) } \\
43.55 \mathrm{I}-0.63 \mathrm{~J}-0.19 \mathrm{~K}\end{array}$} & \multicolumn{3}{|c|}{ Variance-covariance $\left(D^{2}\right)$} & \multirow{2}{*}{$\begin{array}{c}\text { Volume }\left(\mathrm{D}^{3}\right) \\
0.001\end{array}$} \\
\hline & & & $\begin{array}{c}0.007 \\
-0.002 \\
-0.002\end{array}$ & $\begin{array}{c}-0.002 \\
0.005 \\
0.000\end{array}$ & $\begin{array}{c}-0.002 \\
0.000 \\
0.007\end{array}$ & \\
\hline 2. & $44.22-1.37 \times 96$ & $43.54 \mathrm{I}-0.67 \mathrm{~J}-0.13 \mathrm{~K}$ & $\begin{array}{c}0.013 \\
-0.008 \\
0.005\end{array}$ & $\begin{array}{c}-0.008 \\
0.009 \\
-0.006\end{array}$ & $\begin{array}{c}0.005 \\
-0.006 \\
0.007\end{array}$ & 0.001 \\
\hline 3. & $44.24-1.31 \times 97$ & $43.58 \mathrm{I}-0.61 \mathrm{~J}-0.15 \mathrm{~K}$ & $\begin{array}{c}0.014 \\
-0.005 \\
-0.004\end{array}$ & $\begin{array}{c}-0.005 \\
0.006 \\
-0.001\end{array}$ & $\begin{array}{c}-0.004 \\
-0.001 \\
0.006\end{array}$ & 0.001 \\
\hline
\end{tabular}

TABLE 2: Means in conventional and component notation, variance-covariance matrices and $95 \%$ distribution ellipsoid volumes for the posterior surface corneal curvature measurements are indicated.

\begin{tabular}{|c|c|c|c|c|c|c|}
\hline \multirow{3}{*}{$\begin{array}{l}\text { Session } \\
1 .\end{array}$} & \multicolumn{6}{|c|}{ Posterior surface } \\
\hline & \multirow{2}{*}{$\begin{array}{c}\text { Conventional notation (D) } \\
55.32-4.08 \times 93\end{array}$} & \multirow{2}{*}{$\begin{array}{c}\text { Component notation (D) } \\
53.28 \mathrm{I}-2.03 \mathrm{~J}-0.19 \mathrm{~K}\end{array}$} & \multicolumn{3}{|c|}{ Variance-covariance $\left(D^{2}\right)$} & \multirow{2}{*}{$\frac{\text { Volume }\left(\mathrm{D}^{3}\right)}{6.425}$} \\
\hline & & & $\begin{array}{r}0.098 \\
-0.059 \\
-0.012\end{array}$ & $\begin{array}{c}-0.059 \\
0.079 \\
-0.010\end{array}$ & $\begin{array}{c}-0.012 \\
-0.010 \\
0.159\end{array}$ & \\
\hline 2. & $55.63-4.67 \times 91$ & $53.29 \mathrm{I}-2.34 \mathrm{~J}-0.05 \mathrm{~K}$ & $\begin{array}{r}0.090 \\
-0.018 \\
-0.015\end{array}$ & $\begin{array}{c}-0.018 \\
0.116 \\
0.014\end{array}$ & $\begin{array}{l}-0.015 \\
0.014 \\
0.085\end{array}$ & 7.366 \\
\hline 3. & $55.44-3.99 \times 93$ & $53.45 \mathrm{I}-1.99 \mathrm{~J}-0.21 \mathrm{~K}$ & $\begin{array}{r}0.057 \\
0.027 \\
-0.027\end{array}$ & $\begin{array}{c}0.027 \\
0.080 \\
-0.036\end{array}$ & $\begin{array}{c}-0.027 \\
-0.036 \\
0.046\end{array}$ & 2.573 \\
\hline
\end{tabular}




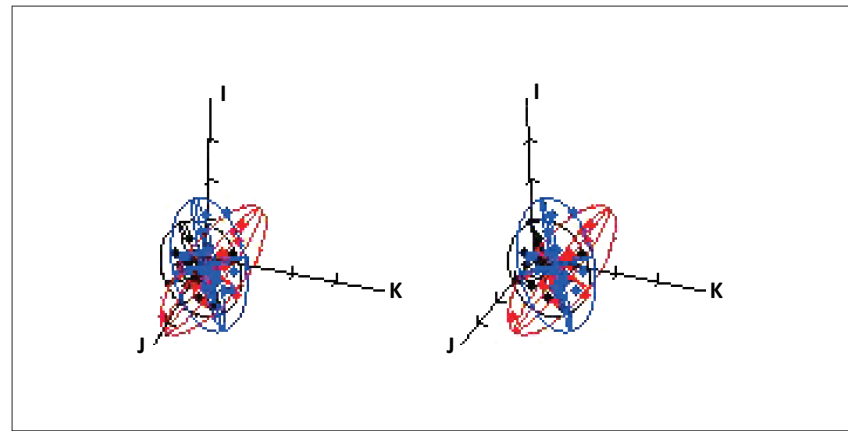

Note: Each data point represents one of 20 Pentacam measurements taken at each session The stereo-pair scatter plot has an axis length of $1 \mathrm{D}$, origin is placed at $44 \mathrm{I} \mathrm{D}$ and the tick interval is $0.25 \mathrm{D}$.

FIGURE 1: Stereo-pair scatter plot with included $95 \%$ distribution ellipsoids for measurements taken of the anterior corneal surface for session one (black data points), session two (red data points) and session three (blue data points).

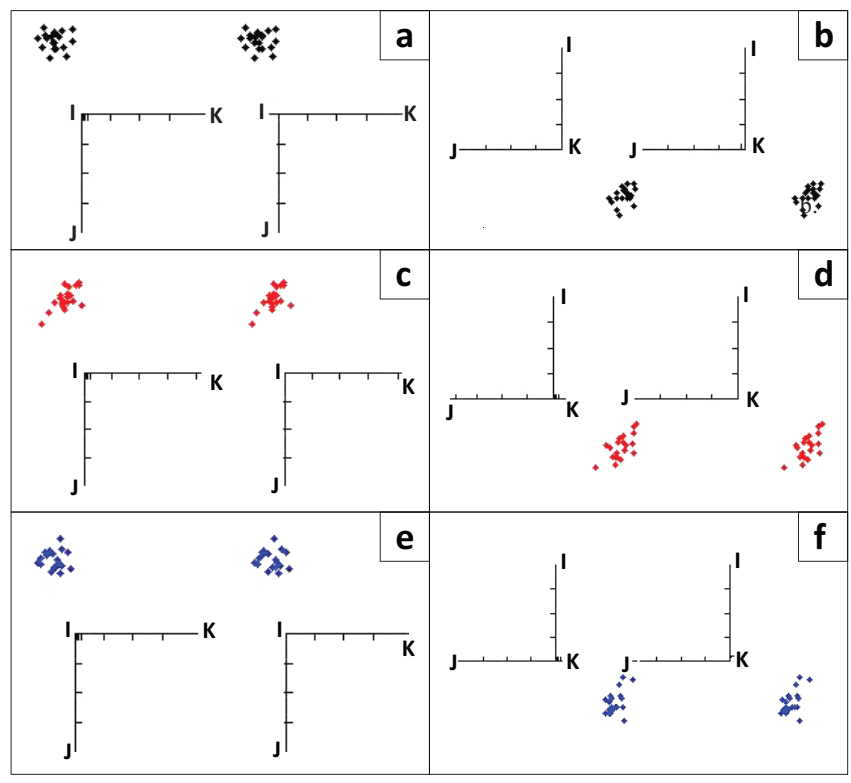

Note: Measurement $s$ are viewed down the stigmatic axis in a, $\mathrm{c}$ and $\mathrm{e}$ and down the oblique antistigmatic axis in b, $d$ and f. The stereo-pair scatter plot has an axis length of $1 D$, origin is placed at $44 \mathrm{I}$ and the tick interval is $0.25 \mathrm{D}$.

FIGURE 2: (a-f) Rotated stereo-pair scatter plots for the anterior surface of the cornea. Each data point represents one of 20 Pentacam measurements taken at the first (black data points), second (red data points) and third (blue data points) measuring session.

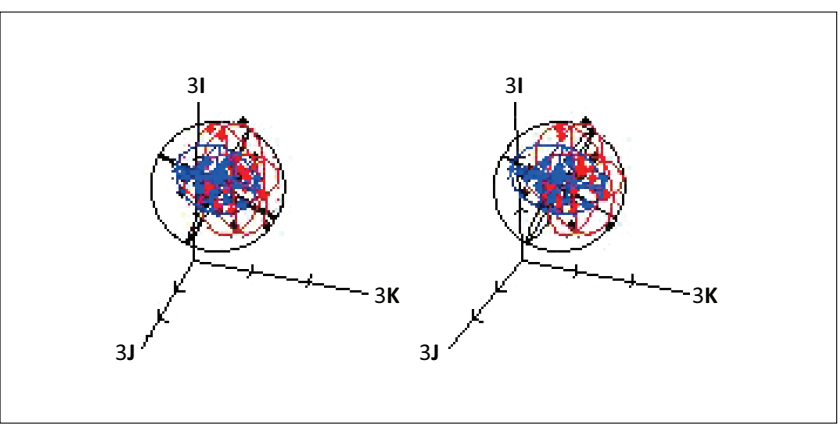

Note: Each data point represents one of 20 Pentacam measurements taken at each session. The stereo-pair scatter plot has an axis length of $3 \mathrm{D}$, origin is placed at $53 \mathrm{I} \mathrm{D}$ and the tick interval is $1 \mathrm{D}$. FIGURE 3: Stereo-pair scatter plot with included $95 \%$ distribution ellipsoids for measurements taken of the posterior corneal surface for session one (black data points), session two (red data points) and session three (blue data points).

axes by $90^{\circ}$ for a view down the stigmatic axis (Figures $2 \mathrm{a}$, c, e and $4 a, c, e)$ and by $180^{\circ}$ degrees for a view down the oblique antistigmatic axis (Figures $2 b, d, f$ and $4 b, d, f)$. This

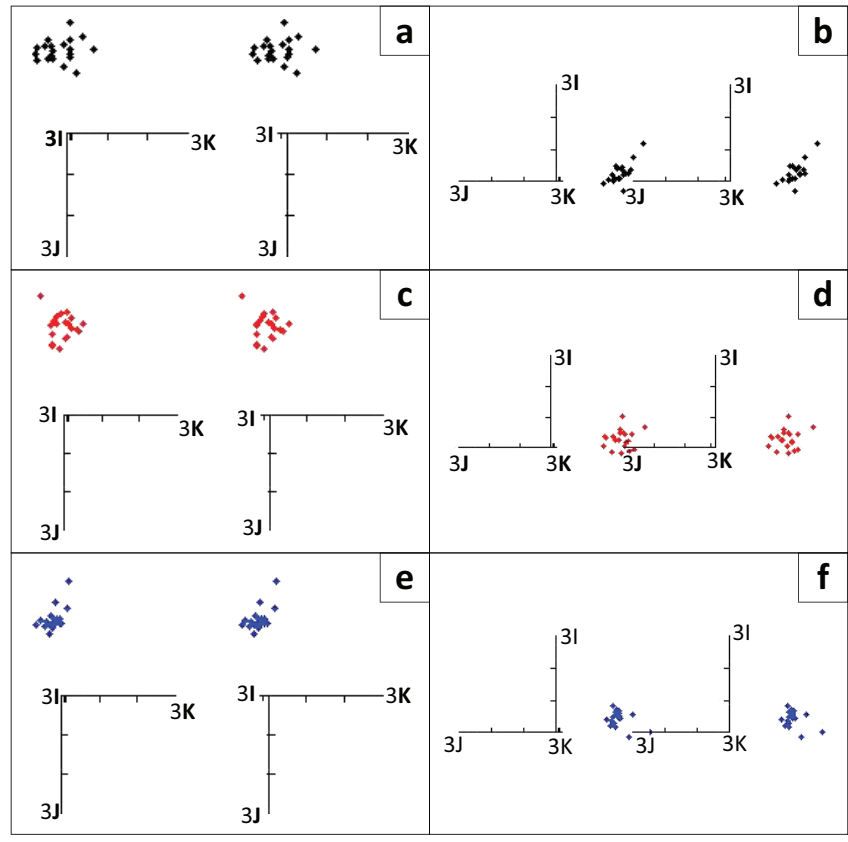

Note: Measurement $s$ are viewed down the stigmatic axis in a, $c$ and $e$ and down the oblique antistigmatic axis in b, $d$ and f. The stereo-pair scatter plot has an axis length of $3 \mathrm{D}$, origin is placed at $53 \mathrm{I}$ and the tick interval is $1 \mathrm{D}$.

FIGURE 4: (a-f) Rotated stereo-pair scatter plots for the posterior surface of the cornea. Each data point represents one of 20 Pentacam measurements taken at the first (black data points), second (red data points) and third (blue data points) measuring session.

will aid readers who are unable to fuse the three dimensional stereo-pairs to view the spread of the data points down the stigmatic $\left(F_{\mathrm{I}} \mathbf{I}\right)$ and oblique antistigmatic $\left(F_{\mathrm{K}} \mathbf{K}\right)$ axes. Each black, red, and bluedata point represents each transformed Sim-K measurement taken in the first, second, and third measurement session respectively. Distribution ellipsoids are included in Figures 1 and 3. The size, shape, and orientation of each distribution ellipsoid provides insight on the variation in intra-session measurements (repeatability) and the comparison of the size, shape, and orientation of the distribution ellipsoids over the different measurement sessions aids in visualising inter-session changes (reproducibility).

\section{Anterior surface corneal curvature}

\section{Repeatability (intra-session) analysis}

Figures 1 and 2 represent keratometric measurements for the anterior corneal surface. The origin of these stereo-pair scatter plots is placed at $44 \mathrm{I} \mathrm{D}$, the axis length is $1 \mathrm{D}$, and the tick interval is $0.25 \mathrm{D}$. One can take note visually, by the small clusters of data points (Figure 1) and quantitatively, by the small distribution ellipsoid volumes (Table 1 ) that there is little variation in the keratometric data for each measuring session. The first measuring session (black data points) has the smallest, tightest cluster of data points (Figures $2 \mathrm{a}$ and $\mathrm{b}$ ) and is therefore probably the most repeatable set of measurements. The second and third measuring sessions also display small clusters of data points with a small amount of stigmatic and antistigmatic (Figures 2c and d) and stigmatic (Figures 2e and f) variation respectively. 


\section{Reproducibility (inter-session) analysis}

As can be seen from Figure 1 most of the data points intersect and form fairly small clusters. The size of all three 95\% distribution ellipsoids is also fairly similar and from Table 1 one can note that all three ellipsoids have the same rounded off volume, that is, $0.001 \mathrm{D}^{3}$. Although the volume is approximately the same for all three distribution ellipsoids, the direction of the spread of measurements varies slightly and Figure 2 makes it easier to see this. The distribution ellipsoid for the first measuring session (black) is more round because of the tighter cluster of data points, however, the distribution ellipsoids for the second (red) and third (blue) sessions are more elliptical. The slightly differing orientation and shape of the distribution ellipsoids can be attributed to the spread of data points along the J-K plane (Figure 2c) and along the stigmatic axis (Figure $2 \mathrm{~d})$ for the second measuring session and along the stigmatic axis (Figure 2f) for the third measuring session.

\section{Posterior surface corneal curvature}

\section{Repeatability (intra-session) analysis}

Figures 3 and 4 represent keratometric measurements for the posterior corneal surface. The origin of these stereo-pair scatter plots is placed at $53 \mathrm{I} \mathrm{D}$, the axis length is $3 \mathrm{D}$, and the tick interval is $1 \mathrm{D}$. As is evident in Figure 3 session three (blue data points) appears to have the least variation with the tightest cluster and smallest distribution ellipsoid volume of $2.573 \mathrm{D}^{3}$ (Table 2) whereas session two (red data points) has the most variation with the most dispersion of data points and the largest distribution ellipsoid volume of $7.366 \mathrm{D}^{3}$.

\section{Reproducibility (inter-session) analysis}

Most of the data points appear to intersect for all three measurement sessions in Figure 3; however, it is over a larger area when compared to the intersection of data points for the anterior corneal surface. One can also note from Figure 3 that the distribution ellipsoids for all three measuring sessions differ in size, shape, and orientation. The black distribution ellipsoid that represents the first measuring session is large $\left(6.425 \mathrm{D}^{3}\right.$, Table 2) and round in shape. When viewing the data points down the stigmatic (Figure 4a) and oblique antistigmatic (Figure $4 b)$ axes one can see that there is both stigmatic and antistigmatic variation. The same can be said for the second measuring session (Figures $4 \mathrm{c}$ and $\mathrm{d}$ ). Although there is also stigmatic and antistigmatic variation in the third measuring session, the amount of variation is less than the two previous measuring sessions as is evident by the tighter cluster of data points (Figures $4 \mathrm{e}$ and $\mathrm{f}$ ) and the smaller distribution ellipsoid volume (Table 2). One can also note the stigmatic and antistigmatic variation quantitatively from the variance-covariance matrices over the three measuring sessions in Table 2.

\section{Hypothesis testing}

Multivariate analysis of variance hypothesis testing done at a 95\% level of confidence was possible because the keratometric data was analysed in its multivariate form. ${ }^{15}$ The null hypothesis was rejected if the test statistic was greater than
TABLE 3: Hypothesis tests done on variance-covariance matrices and means for the anterior and posterior corneal surfaces.

\begin{tabular}{llll}
\hline Test & Hypothesis & Critical value & Test statistic \\
\hline Test 1: Variance- & Anterior corneal surface & $\chi^{2}{ }_{(\alpha, 12)} \approx 21.03$ & $\mu=17.27$ \\
covariance & Posterior corneal surface & $\chi_{(\alpha, 12)}^{2} \approx 21.03$ & $\mu=29.26 \dagger$ \\
Test 2: Means & Anterior corneal surface & $F_{(\alpha, 2,26.5)} \approx 0.18$ & $\theta=0.15$ \\
& Posterior corneal surface & $F_{(\alpha, 2,26.5)} \approx 0.18$ & $\theta=0.27 \dagger$ \\
\hline
\end{tabular}

Note: The null hypothesis is rejected if the test statistic is greater than the critical value. $\dagger$, Indicates rejected hypotheses.

the critical value. ${ }^{21}$ Table 3 provides the test statistics and critical values for the hypothesis tests. The null hypothesis for the first hypothesis test is that variance-covariances are equal and the null hypothesis for the second hypothesis test is that all means are equal. The null hypothesis on variancecovariance is accepted for the anterior corneal surface but rejected for the posterior corneal surface. The hypothesis test on the means had the same outcome.

\section{Discussion}

The limited publications available on the precision of corneal curvature measurements prompted the need for this study. Those studies that are available either confused the meaning of precision or did not analyse the data in a multivariate manner which is necessary when dealing with keratometric data. Laursen et al. ${ }^{9}$ converted Sim-K values into spherical equivalents for their analysis of precision whereas others ${ }^{10,11,12}$ analysed keratometric measurements along the flat and steep meridians separately. Laursen et al. ${ }^{9}$ and Viswanathan et al. ${ }^{10}$ found the Pentacam to have good repeatability and reproducibility for anterior corneal surface measurements. Crawford et al. ${ }^{12}$ had only tested repeatability and found it to be good for anterior corneal surface measurements. Chen and Lam ${ }^{11}$ demonstrated that the Pentacam had good reproducibility for both the anterior and posterior corneal surfaces; however, repeatability was good for all parameters except for the posterior power vector $J_{45}$. Using three dimensional dioptric power space and multivariate statistics Gillan $^{22}$ investigated the repeatability of anterior and posterior corneal curvature in a mildly keratoconic eye and found the anterior corneal surface to exhibit more keratometric variation. This could possibly be related to the irregular anterior corneal surface often associated with keratoconus. To the best of my knowledge this is the first study that has investigated the precision of the Pentacam's keratometric measurements of both the anterior and posterior surfaces of a normal cornea using multivariate statistics which provide a complete and correct analysis of keratometric data.

Figures 1 and 2 represent the three measuring sessions for the anterior corneal curvature. From these two figures one can take note visually that the Pentacam measurements of the anterior corneal curvature are probably both repeatable and reproducible. The small, tight clusters of data points at each measuring session provide evidence of repeatability of the anterior corneal measurements. Reproducibility of the anterior corneal measurements is portrayed by the similarities of the distribution ellipsoid volumes and is further substantiated by the hypothesis test (Table 3) which shows 


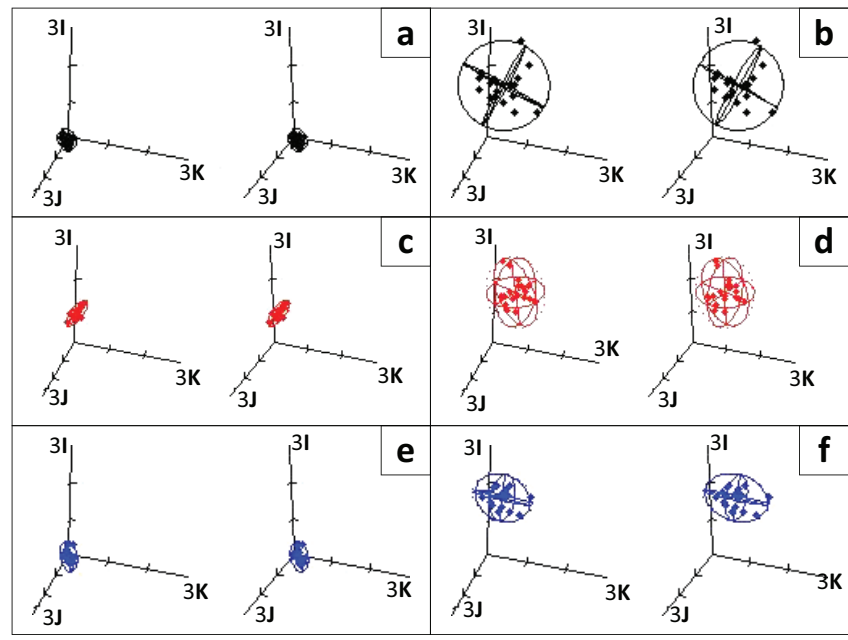

Note: The origin of the stereo-pairs representing the anterior corneal surface $(a, c, e)$ is placed at $44 I D$ and that of the posterior corneal surface $(b, d, f)$ is placed at $531 \mathrm{D}$.

FIGURE 5: (a-f) Rotated stereo-pair scatter plots for the anterior (a, c, e) and posterior $(b, d, f)$ surface of the cornea. All six stereo-pair scatter plots have an axis length of $3 \mathrm{D}$ and the tick interval is $1 \mathrm{D}$.

that the means for all three measuring sessions are statistically similar and hence reproducible.

Unlike the anterior corneal surface, the keratometric measurements of the posterior corneal surface are more variable. Figure 5 shows a direct, side by side comparison of the variation of the anterior and posterior corneal surface at each of the three measuring sessions. In Figure 5 the keratometric data for the anterior corneal surface was plotted on similar axes as the posterior corneal surface measurements to allow for a direct comparison of distribution ellipsoids and spread of data. When compared to the anterior corneal surface measurements (Figures $5 \mathrm{a}, \mathrm{c}$, and e) keratometric data for the posterior corneal surface (Figures $5 b, d$, and $f$ ) appear to be more variable for each of the three measuring sessions as is evident by the larger distribution ellipsoids and is, therefore, probably less repeatable than the measurements taken of the anterior corneal curvature. The reproducibility of Pentacam measurements taken of the posterior corneal surface appears to be poor as is evident in the differing size, shape, and orientation of the representative distribution ellipsoids (Figure 3) and the hypothesis test also shows that the means are statistically different over the three measuring sessions. Although the means are statistically different there is no substantial clinical difference in the means over the three measuring sessions (Table 2). In terms of the anterior corneal surface measurements the results from this study agree with other studies. ${ }^{9,10,11,12}$ As is also evident in this study, Chen and $\mathrm{Lam}^{11}$ found the precision of the posterior corneal surface to be poor.

With the outcomes of any study one should bear in mind the shortcomings. For this particular study the short time over which measurements were taken and using only one subject with a normal cornea could be seen as possible limitations.

\section{Conclusion}

Variation of measurements taken of the posterior corneal surface could possibly be a result of diurnal variation and that of the anterior corneal surface could be a result of blinking, tear film disruption, or diurnal variation, for example. ${ }^{20}$ One would assume that with the anterior corneal surface being vulnerable to both internal and external influences it should display more variation than the posterior corneal surface; however, this is not the case as is evident in Figure 5. It is unknown whether it is operator-instrument influences (such as successive measurements not taken on exactly the same spot) or if it is internal influences (corneal shape change in response to accommodation for example) that make the posterior corneal surface exhibit more variation, ${ }^{23}$ hence further research is required to investigate this complexity.

The Oculus Pentacam appears to have good clinical and statistical precision for keratometric measurements taken of the anterior corneal surface and this is in agreement with other studies done., ${ }^{90,11,12}$ Measurements of the posterior corneal surface appear to be more variable, and although the instrument may not have statistically good precision for keratometric measurements of the posterior corneal surface, it is, however, clinically sound.

\section{Acknowledgements}

I would like to convey my sincere gratitude to WDH Gillan for his guidance through this study and to the fourth-year student who sacrificed precious time to volunteer as my test subject.

\section{Competing interests}

The author declares that she has no financial or personal relationships which may have inappropriately influenced her in writing this article.

\section{References}

1. ISO 5725 (1994), Accuracy (trueness and precision) of measurement methods and result - Part 1: General principles and definitions.

2. McAlinden C, Khadka J, Pesudovs K. Statistical methods for conducting agreement (comparison of clinical tests) and precision (repeatability or reproducibility) studies in optometry and ophthalmology. Ophthal Physiol Opt. 2011;31:330-338.

3. Jain R. Pentacam: Principle and clinical applications. J Curr Glau Prac. 2009;3:20-32.

4. Pentacam instrument manual: Measurement and evaluation system for the anterior segment of the eye. Wetzlar, Germany: Oculus 70700; 2005.

5. Doménech B, Mas D, Ronda E, Pérez J, Espinosa J, Illueca C. Repeatability and concordance of the Pentacam system. Comparative study of corneal parameters measured with Pentacam and Atlas. Opt Pura Apl. 2009;42:51-60.

6. Fu J, Wang $\mathrm{X}$, Li S, Wu G, Wang N. Comparative study of anterior segment measurement with Pentacam and anterior segment optical coherence tomography. Can J Ophthalmol. 2010;45:627-631.

7. de Sanctis U, Missolungi A, Mutani B, Richiardi L, Grignolo FM. Reproducibility and repeatability of central corneal thickness measurement in keratoconus using and repeatability of central corneal thickness measurement in keratoconus using 2007;144:712-718.

8. Martina R, Jonuscheit S, Rio-Cristobal A, Doughtyc MJ. Repeatability of Pentacam peripheral corneal thickness measurements. Cont Lens Anterior Eye. 2015;38: 424-429. http://dx.doi.org/10.1016/j.clae.2015.05.001

9. Laursen JVN, Jeppesen P, Olsen T. Precision of 5 different keratometry devices. Int Ophthalmol. 2015;36(1):17-20. http://dx.doi.org/10.1007/s10792-015-0069-3

10. Viswanathan D, Kumar NL, Males JJ, Graham SL. Comparative analysis of corneal measurements obtained from a Scheimpflug camera and an integrated Placido-optical coherence tomography device in normal and keratoconic eyes. Acta Ophthalmologica. 2015;93:488-494.

11. Chen D, Lam AKC. Reliability and repeatability of the Pentacam on corneal curvatures. Clin Exp Optom. 2009;92:110-118.

12. Crawford AZ, Patel DV, McGhee CNJ. Comparison and repeatability of keratometric and corneal power measurements obtained by Orbscan II, Pentacam, and Galilei corneal tomography systems. Am J Ophthalmol. 2013;156:53-60. 
13. Harris WF. The matrix representation of dioptric power. Part 1: An introduction. S Afr Optom. 1988;47:19-23.

14. Harris WF. Graphical representation of symmetric dioptric power and the concepts of stigmatic, astigmatic and antistigmatic power. S Afr Optom. 2002;61: 126-134.

15. Harris WF. Testing hypotheses on dioptric power. Opt Vis Sci. 1992;69:835-845.

16. Harris WF, Malan DJ, Rubin A. Ellipsoidal confidence regions for mean refractive status. Optom Vis Sci. 1991;68:950-953.

17. Harris WF. Representation of dioptric power in Euclidian 3-space. Ophthal Physiol Opt. 1991;11:130-136

18. Malan DJ. Dioptric power data analysis. S Afr Optom. 1993;52:84-90.
19. Malan DJ. The excess of objective automatic refraction over subjective clinical refraction: Methods of analysis, results [Master's degree dissertation] Johannesburg, South Africa: Rand Afrikaans University; 1994

20. Chetty E. The short-term effects of polymethyl methacrylate and rigid gas permeable contact lens wear on keratometric behaviour [Master's degree dissertation]. South Africa: University of Johannesburg; 2011.

21. Heck DL. Charts of some upper percentage points of the distribution of the largest characteristic root. Ann Math Statist. 1960;31:625-642.

22. Gillan WDH. Variation in surface power and thickness of a moderately keratoconic cornea. S Afr Optom. 2008;67:4-10.

23. Rubin A, Mathebula SD. Global and local contributions to surface curvature of healthy corneas. S Afr Optom. 2012;71:146-158. 